\title{
DETERMINACIÓN DE LAS RESERVAS DE CARBONO DE LA BIOMASA AÉREA, EN DIFERENTES SISTEMAS DE USO DE LA TIERRA EN SAN MARTÍN, PERU
}

\section{DETERMINATION OF CARBON RESERVES OF THE AERIAL BIOMASS IN DIFFERENT LAND USE SYSTEMS IN SAN MARTIN, PERU}

\author{
Tatiana Lapeyre ${ }^{1}$, Julio Alegre ${ }^{2}$ y Luis Arévalo ${ }^{3}$
}

\begin{abstract}
Resumen
Se determinó la biomasa aérea en diferentes sistemas de uso de la tierra en la región de San Martín-Perú, con la finalidad de conocer el potencial de captura de carbono. Los sistemas de uso de la tierra evaluados fueron: Bosque primario, Bosque secundario de diferentes edades, sistemas agrícolas locales maíz (Zea maiz), arroz (Oriza Sativa), pastos (Brachiaria) y sistemas agroforestales con café (Coffea arabica) bajo sombra y cacao (Cacao sp.). También se comparó este potencial con otros sistemas de uso de la tierra de otras regiones del Perú y se monitoreó la pérdida de reservas de carbono después del corte de la foresta y su reemplazo por cultivos. En cada uno de éstos sistemas se establecieron al azar cinco transectos donde se evaluó la biomasa arbórea. Dentro de éstos transectos se establecieron cuadrados también al azar para cuantificar la biomasa herbácea y la biomasa de hojarasca. El carbono total en el bosque primario fue de $485 \mathrm{tm}$ $\mathrm{C}$ ha-1, superando ampliamente las reservas del bosque secundario de 50 años y de bosque descremado de 20 años. Con relación al bosque primario se observa una reducción de reservas en más de $50 \%$ del bosque secundario de 50 años (234 tm C ha-1). El bosque descremado de 20 años perdió más del $80 \%$ de reservas $(62 \mathrm{tm} \mathrm{C}$ ha-1). El nivel de reservas de carbono en la biomasa de hojarasca de los sistemas boscosos, no es significativo al compararlo con el total de las reservas de carbono de la biomasa aérea; sin embargo si es significativo para sistemas agroforestales. Los sistemas agroforestales secuestraron entre 19 a $47 \mathrm{tm} \mathrm{C}$ ha-1, dependiendo de la cantidad de especies forestales, tipo de cultivo, edad y tipo de suelo y recuperan el potencial de captura en forma productiva. Los sistemas agrícolas capturaron poco C (5 tm C ha-1), además generan fugas de gases efecto invernadero (GEI) cuando se usan agroquímicos y quema de rastrojos, entre otros.
\end{abstract}

Palabras clave: carbono, secuestramiento, sistemas de uso de la tierra, agroforestería.

\begin{abstract}
Carbon (C) reserves of the aerial biomass were determined, to evaluate the potential of $\mathrm{C}$ captures in different land uses in the region of, San Martin, Perú. The land use systems (LUS) were: Primary forest, secondary forest of different ages, traditional agricultural maize systems (Zea maiz), rice (Oriza Sativa), pastures (Brachiaria) and coffee agroforestry systems (Coffea arabica) under shade and cocoa (Cacao sp.) also under shade. These LUS were compared with other LUS of other regions of Perú and the loss of $\mathrm{C}$ reserves were monitored after land clearing of the forest for cropping. Five random transects for each land use system and within each of these transects; tree and herbaceous biomass and litter biomass were monitored.

Total C in primary forest was $485 \mathrm{tm} \mathrm{C}$ ha-1, much higher than the carbon reserves of the 50 years secondary forest (234 tm C ha-1 which is 50\% lost) and the 20 years logged forest (60 $485 \mathrm{tm} \mathrm{C}$ ha- 1 which is $80 \%$ lost). The level of $\mathrm{C}$ reserves in the biomass litter of the wooded systems is not significant when compared with the total of the $\mathrm{C}$ reserves of the aerial biomass; nevertheless it is significant for agroforestry systems. The agroforestry systems sequestered between 19 a $47 \mathrm{tm} \mathrm{C}$ ha- 1 and varied in tree species, type and age of crop, soil class and recovered the $\mathrm{C}$ capture potential in a productive way. The cropping systems sequestered low levels of $\mathrm{C}\left(5 \mathrm{tm} \mathrm{C} \mathrm{ha}^{-1}\right)$ and generated greenhouse gas emissions (GGE) when burning or over use of agrochemicals or other inputs, are imployed.
\end{abstract}

Key words: carbon, sequestering, land use systems, agroforestry.

\section{Introducción}

El carbono es un componente esencial de todos los seres vivos. Existe en su mayor parte como dióxido de carbono en la atmósfera, los océanos y los combustibles fósiles (carbón, petróleo y otros hidrocarburos). El dióxido de carbono en la atmósfera es absorbido por las plantas y convertido en carbohidratos y tejidos a través del proceso de fotosíntesis, como parte del ciclo del carbono. 
Los bosques tropicales desempeñan un papel vital en el ciclo global del carbono. Los bosques tropicales, al cubrir un área extensa y ser ecosistemas extremadamente dinámicos, regulan la cantidad de dióxido de carbono en la atmósfera mediante la fijación del mismo y su reincorporación al ciclo normal (Dixon, 1995; Gralb et al., 2001).

En los últimos años The International Centre for Research in Agroforestry (ICRAF), ahora "The World Agroforestry Centre" ha desarrollado estudios que evalúa las reservas de carbono en diferentes sistemas de uso de la tierra, en dos sitios de los trópicos húmedos del Perú y un sitio de trópico semi-seco dentro de un ecosistema de montañas.

La primera área de estudio está ubicada en la región amazónica, en la provincia de Alto Amazonas, distrito de Yurimaguas a una altura de $180 \mathrm{msnm}$ y con precipitaciones anuales de $2200 \mathrm{~mm}$ y una temperatura promedio de $26^{\circ} \mathrm{C}$. El suelo es un Ultisol típico, silicio Iso-hipertérmico, la textura de los suelos son franco arenosos con porcentajes de arcilla no mayor de $20 \%$ en los primeros $15 \mathrm{~cm}$ de profundidad. El nitrógeno y la materia orgánica son bajos con alta acidez y con bajos niveles de cationes y fósforo y alta saturación de aluminio.

La segunda área de estudio, está ubicada en la Región de Ucayali, Pucallpa, Perú, también en la región amazónica, a una altura de $250 \mathrm{msnm}$ y con precipitaciones anuales que van desde los $1800 \mathrm{~mm}$ hasta los $3500 \mathrm{~mm}$ por año y una temperatura promedio de $25^{\circ} \mathrm{C}$.

Los sistemas de uso de la tierra estudiados en la zona de Yurimaguas fueron los de barbechos mejorados de ciclo corto. En Pucallpa se estudiaron sistemas de barbechos de ciclo corto así como un bosque de mas de 40 años con la madera valiosa ligeramente extraída y bosques secundarios de 3.5 y 25 años. Otros sistemas evaluados fueron los pastos naturales degradados de más de 30 años.

En el departamento de San Martín, la superficie total del área boscosa es de 3553642 ha $(72.45 \%$ del bosque original), estas áreas se encuentran en zonas montañosas. La superficie deforestada en el año 1990 fue de 1351158 ha, muchas de éstas áreas han sido usadas para la producción de cultivos anuales, pastos, sistemas perennes y en algunos casos agroforestería. Algunos de estos sistemas de uso de la tierra han reducido la capacidad de capturar o fijar carbono.

Los objetivos de la investigación fueron:

- Cuantificar las reservas de carbono de la biomasa aérea en diferentes sistemas de uso de la tierra en la localidad de San Martín.

- Determinar la proporción relativa del aporte de carbono en cada componente del sistema.

- Discutir algunas implicancias ambientales que puedan tener éstas reservas de carbono por la deforestación.

\section{Ubicación de los sistemas}

Los sistemas evaluados fueron localizados en el Departamento de San Martín en las provincias de Lamas y San Martín. Geopolíticamente el sistema de bosque primario se encuentra ubicado en la provincia de Lamas, distrito de Tabaloso; el sistema de bosque secundario de 50 años se encuentra ubicado en la provincia de San Martín, distrito de La Banda de Shilcayo; el sistema de bosque secundario de 20 años se encuentra ubicado en la provincia de San Martín, distrito de La Banda de Shilcayo; los sistemas agrícolas (arroz, maíz) y el sistema de pastos mejorados se encuentran en la provincia de San Martín, distrito Juan Guerra; el sistema agroforestal de café se encuentra en la provincia de Lamas, distrito Zapatero; y el sistema agroforestal de cacao se encuentra ubicado en la provincia San Martín, distrito Tarapoto.

\section{DESCRIPCIÓN DE LOS SISTEMAS EVALUADOS EN SAN MARTÍN.}

Sistema de Bosque Primario

El bosque primario evaluado pertenece a la comunidad de Ungurahuy, quienes poseen un total de $6000 \mathrm{ha}$, el área evaluada fue de 4 ha. Se encuentra a una altura de 1193 msnm, con gran variedad de especies y abundante estrato de sotobosque, presenta laderas con fuerte pendiente desde $50 \%$. En éste ecosistema se puede observar árboles con diámetro a la altura del pecho (dap) $>30 \mathrm{~cm}$.

No se ha realizado aprovechamiento de especies en esta área, y se observa especies muertas y caídas de forma natural.

Se determina a éste bosque como, Bosque húmedo de montañas, por sus características fisiográficas, así mismo presenta una densa vegetación, con especies arbóreas como quina quina (Zschokka sp.), requia (Guarea guidonia), cumala (Virola sp.), lagarto caspi (Callophylum brasilense), moena canela (Nectándra sp.), shimbillo (Inga sp.) abundantes especies de palmeras (360/ha) principalmente la huacrapoma utilizada para parquet. No se observa gran número de especies epífitas, éste bosque tiene potencial maderable, tal como podemos observar por las especies encontradas.

El bosque evaluado presenta diversidad de especies arbóreas, muchas de la cuales son comerciales y otras utilizadas como medicina natural; con relación a especies de fauna silvestre, según lo indicado por pobladores del lugar se encuentran variedades de ofidios como la shushupe (Lachesis muta), boas comunes (Contrictor contrictor), aves como el tucán (Ramphastus momiles cuvieri), el carpintero (Geophlocus lincatus), el pihuicho (Psilopsiagon aurifrons) y lechuzas (Ninox sp.), con relación a mamíferos hay presencia de ronsoco (Hidrochoerus hridrochoeris), coto mono (Alovata seniculus), entre otros. 


\section{Sistema de Bosque Secundario- 50años}

El bosque secundario de 50 años evaluado, forma parte del cerro escalera, que tiene una característica climática regional determinante en el clima local, ya que intercepta las masas de aire húmedo provenientes de la Amazonía. La temperatura hasta los $1000 \mathrm{msnm}$ se sitúa alrededor de $\operatorname{los} 25^{\circ} \mathrm{C}$, a partir de los 1000 hasta los $2000 \mathrm{msnm}$, la temperatura disminuye hasta $\operatorname{los} 22{ }^{\circ} \mathrm{C}$. El Cerro Escalera forma parte de la cadena de montañas Cahuapanas que se extienden longitudinalmente al noreste de la ciudad de Tarapoto, esta cadena presenta la cadena mas joven en Andes Orientales y concentra la cadena de montañas Cerros Azules del Biabo y las montañas del Bosque de protección Nacional Alto Mayo y Cordillera Colán.

El área evaluada fue de 4 hectáreas y es localizada sobre los $700 \mathrm{msnm}$; hace aproximadamente 50 años, que ésta área es protegida y no presenta aprovechamiento de especies, el relieve del lugar evaluado es suave- ondulado con pendientes de máximo $50 \%$. Se determinaron especies como tortilla caspi (Psychotria sp.), moena (Aniba amazónica), entre otras.

\section{Sistema de Bosque Secundario-20 años}

El sistema de bosque secundario de 20 años, se localiza en el distrito de La banda del Shilcayo, en un área que se ha desarrollado luego de un aprovechamiento selectivo de especies comerciales y del uso de leña por la intervención de pobladores cercanos al área.

Se observa un ecosistema en recuperación, con la mayoría de especies forestales por debajo de los 30 $\mathrm{cm}$ dap, abundante sotobosque y lianas. De acuerdo a lo mencionado por el propietario del área el sistema no ha sido intervenido en los últimos 20 años, se pudo apreciar áreas con especies menores a $10 \mathrm{~cm}$ de dap, sin embargo, por lo observado no se considera que el sistema no ha sido intervenido en los últimos 20 años y por ello se le ha determinado como un sistema descremado.

El relieve es abrupto en algunos sectores, con pendientes mayores a $100 \%$, pero en la mayoría del área predomina una pendiente menor al 50\%. Entre las principales especies encontradas tenemos: shimbillo (Inga sp.), cetico (Cecropia peltandra), ana caspi (Apulcia molares).

\section{Sistema de arroz (Oriza sativa)}

El sistema de arroz se localiza en la Estación Experimental El Porvenir del Instituto de Investigación Agraria, tiene un tiempo de siembra de 70 días y un área de 5.5 ha.

La preparación del terreno la realizan mediante un arado antes de la siembra, el lote evaluado tiene como fin la producción de semilla e investigación y es de la variedad capirona. Se realizan dos campañas al año, la primera de Enero a Julio y la segunda de Agosto a Diciembre.
El sistema es rotativo, con pozas de descanso de 1 a 2 meses y, la fertilización la realizan 150-60-60 de $\mathrm{N}, \quad \mathrm{P}_{2} \mathrm{O}_{5}$ y $\mathrm{K}_{2} \mathrm{O}$ respectivamente; el fósforo se incorpora en dos partes de 75 y 75 . El deshierbo se realiza con herbicida pre-emergente Machete y/o Saturn, después del transplante con herbicida postemergente a los 15-20 días.

\section{Sistema de maíz (Zea maiz)}

El sistema de maíz se localiza, también en la Estación Experimental El Porvenir del Instituto de Investigación Agraria, tiene un tiempo de siembra de 90 días y un área de 8 ha, la distancia de siembra de $80 \times 50$, dos plantas por golpe, 51 plantas / ha.

La siembra se mantiene con lluvias del lugar, y se obtiene 4.5 ton/ha. Se aplica herbicida preemergente Atracina 1.5 litros /ha, una sola aplicación antes de la siembra, y Fasta para el control de cogollero 1 litro / ha.

La fertilización con N-P-K, se realiza con las siguientes proporciones 120,80 y 60 de de $\mathrm{N}, \mathrm{P}_{2} \mathrm{O}_{5}$ y $\mathrm{K}_{2} \mathrm{O}$ respectivamente. El nitrógeno se aplica fraccionado, 60 en la siembra y 60 en la floración. Todo material de cosecha es incorporado nuevamente al terreno, no se realiza quema y el fin de la producción es la semilla la cual se obtiene a los 120 días de sembrado.

\section{Sistema de pastos manejados}

Este sistema pertenece a la Estación Experimental El Porvenir, el área evaluada es de 2 ha. La especie Brachiaria brisantha, especie plantada desde 1996, son pasturas sin abonamiento, la siembra se realiza con preparación mecánica y siembra manual; posee una densidad de siembra de 1x $60 \mathrm{~cm}$, se produce la floración en un promedio de 140 días, y se realiza fertilización orgánica por ovinos.

El pasto evaluado tiene 3 meses con una altura promedio de $80 \mathrm{~cm}$. El pastoreo se hace con 24-30 animales cada 15 o 20 días. Se realiza una quema controlada en el área si existen problemas de garrapatas. Los niveles de fertilización son de 45,45 y 30 de N-P-K. y se obtiene $15 \mathrm{~kg}$ de semillas por ha y $46-55 \mathrm{~kg} / \mathrm{ha}$ de forraje verde.

\section{Sistema de café (Coffea arbica) - guaba (Inga edulis)}

El sistema Agroforestal café (Coffea arbica) guaba (Inga edulis) se localiza en la localidad de Nuevo Mundo, con trocha carrozable de acceso a la parcela. La edad del sistema es de 04 años combinada con especies de guaba con un total de 7 ha plantadas a un distanciamiento de $1.5 \times 1.5 \mathrm{~m}$.

La época de cosecha son los meses de Marzo a Junio La producción promedio es de 15 quintales por hectárea.

No se usa abono para el mantenimiento y los deshierbo son manuales con machete. Se utiliza sin embargo el insecticida Thiodan para la broca del café. La poda de las dos especies del sistema (el café y la 
guaba) se realiza durante los meses de Agosto a Octubre.

El terreno no es inundable y posee una pendiente de $60 \%$ en los lugares más inclinados. Presenta solo a la guaba, como especie forestal que otorga sombra al café y como recicladora de nutrientes. El área basal de ésta especie es de 1.92 $\mathrm{m}^{2} /$ ha y se estima en 280 árboles por /ha.

\section{Sistema de cacao (Cacao sp.)}

El Sistema de cacao ubicado en la localidad de Cachiyacu, cercano al río Cumbaza y a la ciudad de Tarapoto sobre un suelo rojizo con textura franco arcilloso y estructura moderada y subangular, con pendientes de $30 \%$ y no inundable. La precipitación localizada es de 800 a $1000 \mathrm{~mm}$, con una temperatura promedio de $25^{\circ} \mathrm{C}$ y una humedad relativa de $70 \%$.

La edad de la plantación es de 15 años con especies forestales entre los 15 y 20 años. El área basal total de especies forestales es de $5.71 \mathrm{~m}^{2} / \mathrm{ha}$, estimándose entre las principales especies: 30 árboles de guaba/ha (Inga edulis), 60 árboles/ha de pumaquiro (Simira sp), 40 árboles/ha de bolaina blanca (Guazuma crinita), 20 árboles/ha de bolaina negra (Guazuma ulmifolia), 20 árboles/ha de shimbillo blanco (Inga sp.).

El área de la plantación es de 3 ha. El cacao fructifica al tercer año, y en los meses de mayor cosecha son entre Abril y Agosto y los meses con menor cosecha, Setiembre a Diciembre produciendo en promedio 350 $\mathrm{kg}$ por hectárea.

El mantenimiento se realiza mediante podas regulares tanto para el cacao como para las especies forestales y el desmalezado mediante machete, no se aplica insecticidas, tampoco abonos (ya sea químicos u orgánicos). La densidad de la plantación es de 4 x 4 metros.

\section{Materiales y métodos}

Para la estimación del carbono contenido en los diferentes sistemas de uso de la tierra, se seleccionaron los sistemas más frecuentes y de mayor importancia en el Departamento de San Martín, hoy región San Martín, de acuerdo a lo verificado en campo y a los objetivos de comparación.

En los sistemas boscosos el inventario exploratorio forestal solo tiene el propósito de estimar el volumen de madera (expresan el volumen en los troncos principales con valor comercial) y no es suficiente para la estimación de la cantidad de carbono, ya que se ignora el volumen de los componentes de los árboles sin valor comercial y otras fuentes de carbono en el ecosistema.

La determinación del número de muestras de cada uno de los sistemas se realiza con un coeficiente de variación del $30 \%$ para sistemas boscosos y $20 \%$ para sistemas agrícolas. El 20\% de error de la muestra considerando que sería a nivel exploratorio, es el error utilizado en sistemas forestales para la realización de inventarios, en los actuales Planes de Manejo Forestal que vienen desarrollando, supervisados por el Instituto Nacional de Recursos Naturales.

Fórmula: $\mathrm{n}=\left[\left(\mathrm{T}^{2} \mathrm{CV}\right) / \mathrm{E}\right]^{2}$.

La comparación de las evaluaciones se realizó mediante el diagrama de cajas (boxplot). Éste es un gráfico simple ya que se realiza básicamente con cinco números, en donde se observa en forma clara la distribución de los datos, dispersión de las medias y sus principales características. También permite comparar en forma simultánea diversos conjuntos de datos.

Se utiliza como herramienta visual para ilustrar los datos, para estudiar simetría, colas y supuestos sobre la distribución y para comparar diferentes poblaciones (Juran \& Gryna, 1988).

El gráfico contiene un rectángulo, orientado con el sistema de coordenadas tal que el eje vertical tiene la misma escala del conjunto de datos, la parte superior y la inferior del rectángulo coinciden con el tercer cuartel y el primer cuartel de los datos. Esta caja se divide con una línea horizontal a nivel de la mediana, y una línea vertical (un bigote) que se extiende desde la mitad de la parte superior de la caja hasta la mayor observación de los datos si se encuentran dentro de un paso, definiendo un "paso" como 1.5 veces el rango intercuartil; igual se hace en la parte inferior de la caja.

En cada uno de los transectos o muestras de los sistemas se realiza la metodología de evaluación presentada en el siguiente ítem, tratando de considerar todos los tipos de sumideros aéreos de los sistemas (árboles, arbustos, herbáceas y hojarascas).

Metodología de la evaluación

La metodología de evaluación que se utilizó fue desarrollada por el ICRAF siguiendo los procedimientos del Manual de determinación de las reservas totales de carbono en los diferentes sistemas de uso de la tierra (Arévalo et al., 2003). Esta metodología se basa en la determinación de la biomasa total mediante el uso de ecuaciones alométricas si son árboles y en la toma de muestras en forma disturbativa del sotobosque, cultivos, pastos etc. así como la hojarasca (Arévalo et al., 2003; Woomer et al., 2000).

\section{Resultados y discusión}

\section{Número de individuos y área basal}

En el bosque primario se encontró el siguiente número de individuos/ha y área basal /ha, en cada una de las siguientes clases diamétricas (dap): $<10 \mathrm{~cm} 1460$ ind/ha y $3.58 \mathrm{~m}^{2} / \mathrm{ha} ; 10-20 \mathrm{~cm} \quad 600 \mathrm{ind} / \mathrm{ha} \mathrm{y}$ $8.61 \mathrm{~m}^{2} / \mathrm{ha} ; 20-30 \mathrm{~cm} 60$ ind/ha y $2.53 \mathrm{~m}^{2} / \mathrm{ha} ;>30 \mathrm{~cm}$ $120 \mathrm{ind} / \mathrm{ha} \mathrm{y} 24.6 \mathrm{~m}^{2} \mathrm{ha}^{-1} \mathrm{~m}^{2} \mathrm{ha}^{-1}$. 
Siendo las siguientes especies las más abundantes y dominantes con dap menores a $30 \mathrm{~cm}$ : shimbillo (Inga sp.) $300 \mathrm{ind} /$ ha y $2.29 \mathrm{~m}^{2} \mathrm{ha}^{-1}$; batajuco (n.i) 240 ind/ha y $1.32 \mathrm{~m}^{2} \mathrm{ha}^{-1}$, moena (Nectándra sp.) 140 ind/ha y $1.09 \mathrm{~m}^{2} \mathrm{ha}^{-1}$, mullaquilla (n.i) $240 \mathrm{ind} / \mathrm{ha} \mathrm{y}$ $\mathrm{m}^{2} \mathrm{ha}^{-1}$, shamoja (Macroenemum sp.) 140ind/ha $\mathrm{y}$ $0.46 \mathrm{~m}^{2} \mathrm{ha}^{-1}$. Las especies más abundantes $\mathrm{y}$ dominantes mayores a $30 \mathrm{~cm}$ de dap: cumala (Virola sebifera) $20 \mathrm{ind} / \mathrm{ha}$ y $4.36 \mathrm{~m}^{2} \mathrm{ha}^{-1}$, leche caspi (Couma macrocarpa) 8 ind/ha y $3.08 \mathrm{~m}^{2} \mathrm{ha}^{-1}$, quina quina (Zschokka so ) $16 \mathrm{ind} / \mathrm{ha}$ y $2.74 \mathrm{~m}^{2} \mathrm{ha}^{-1}$ ), shimbillo (Inga sp.) $16 \mathrm{ind} / \mathrm{ha}$ y $2.28 \mathrm{~m}^{2} \mathrm{ha}^{-1}$, moena (Ocotea sp.) $8 \mathrm{ind} /$ ha y $0.77 \mathrm{~m}^{2} \mathrm{ha}^{-1}$.

En el bosque secundario de 50 años se encontró el siguiente número de individuos/ha y área basal /ha, en cada una de las clases diamétricas (dap): $<10 \mathrm{~cm}$. $1780 \mathrm{ind} / \mathrm{ha} \mathrm{y} 4.47 \mathrm{~m}^{2} \mathrm{ha}^{-1} ; 10-20 \mathrm{~cm} 340 \mathrm{ind} / \mathrm{ha} \mathrm{y}$ $4.96 \mathrm{~m}^{2} \mathrm{ha}^{-1} ; 20-30 \mathrm{~cm}$ 340ind/ha- $4.96 \mathrm{~m}^{2} \mathrm{ha}^{-1} ;>30$ $\mathrm{cm} 100 \mathrm{ind} /$ ha y $20.82 \mathrm{~m}^{2} \mathrm{ha}^{-1}$.

Siendo las siguientes especies las más abundantes y dominantes menores a $30 \mathrm{~cm}$ de dap: tortilla caspi (Psychotria sp.) $200 \mathrm{ind} / \mathrm{ha}$ y $7.90 \mathrm{~m}^{2} \mathrm{ha}^{-1}$; mapichi $220 \mathrm{ind} / \mathrm{ha}$ y $1.78 \mathrm{~m}^{2} \mathrm{ha}^{-1}$,tangarana (Triplaris peruviana) $140 \mathrm{ind} / \mathrm{ha}$ y $1.12 \mathrm{~m}^{2} \mathrm{ha}^{-1}$, moena amarilla (Aniba amazónica) 260ind/ha y $0.66 \mathrm{~m}^{2} \mathrm{ha}^{-1}$. Las especies más abundantes y dominantes mayores a 30 $\mathrm{cm}$ : pino chuncho (Schizolobium sp.) 8 ind/ha y 3.00 $\mathrm{m}^{2} \mathrm{ha}^{-1}$, catahua (Hura crepitans) 8 ind/ha y $2.04 \mathrm{~m}^{2}$ $\mathrm{ha}^{-1}$, fapina (Cupania latifolia) 8 ind/ha y $1.90 \mathrm{~m}^{2} \mathrm{ha}^{-1}$ entre otras.

En el bosque secundario de 20 años se encontró el siguiente número de individuos/ha y área basal /ha, en cada una de las clases diamétricas: $<10 \mathrm{~cm} 1470$ ind/ha y $2.96 \mathrm{~m}^{2} \mathrm{ha}^{-1} ; 10-20 \mathrm{~cm} 320 \mathrm{ind} / \mathrm{ha}$ y $4.65 \mathrm{~m}^{2}$ $\mathrm{ha}^{-1} ; 20-30 \mathrm{~cm} 140 \mathrm{ind} / \mathrm{ha}$ y $6.85 \mathrm{~m}^{2} \mathrm{ha}^{-1} ;>30 \mathrm{~cm} 20$ ind/ha y $1.89 \mathrm{~m}^{2} \mathrm{ha}^{-1}$. Las siguientes especies las más abundantes y dominantes menores a $30 \mathrm{~cm}$ : cetico (Cecropia peltandra) 140 ind/ha y $2.57 \mathrm{~m}^{2} \mathrm{ha}^{-1}$; estaca caspi (n.i) $60 \mathrm{ind} /$ ha y $1.97 \mathrm{~m}^{2} \mathrm{ha}^{-1}$, junjulí (Persea coerulea) $20 \mathrm{ind} / \mathrm{ha}$ y $1.02 \mathrm{~m}^{2} \mathrm{ha}^{-1}$, bellaco caspi (Himatanthsu sucumba) $120 \mathrm{ind} / \mathrm{ha}$ y $0.62 \mathrm{~m}^{2} \mathrm{ha}^{-1}$, entre otros. La única especie mayor a $30 \mathrm{~cm}$ encontrada fue: moena (Aniba amazónica) $20 \mathrm{ind} / \mathrm{ha}$ y $1.90 \mathrm{~m}^{2} \mathrm{ha}^{-1}$.

\section{Reservas de carbono}

Es claro que los sistemas permanentes y con mayor crecimiento presentan los valores más altos de acumulación de Carbono. El Bosque primario contiene un promedio de $485 \mathrm{tm} \mathrm{C} \mathrm{ha}^{-1}$ (100\%), valor que se reduce drásticamente si éste bosque se deforesta y quema para plantar cultivos anuales en las mismas áreas llegando a valores muy bajos de menos de $5 \mathrm{tm} \mathrm{C} \mathrm{ha}^{-1}(1 \%)$. Recuperar el estado inicial de las reservas del bosque primario tomará muchos años, tal como se puede apreciar con el bosque secundario de 50 años que alcanza solo un $48 \%$ de lo que se tenía en reservas el bosque primario (Figura 1).

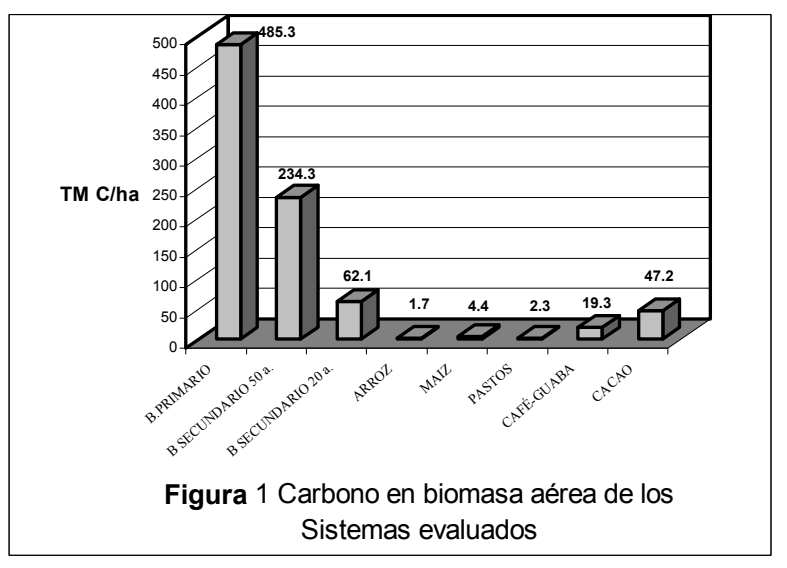

Hay que destacar que no se podrá observar una recuperación significativa de los niveles de captura de carbono si estos sistemas son continuamente perturbados o descremados (extracción selectiva de madera), además de disminuir significativamente su biodiversidad, tal como se observa en el bosque secundario de 20 años ya descremado que contó con solo con $13 \%$ de lo capturado por el bosque primario.

El sistema de café-guaba, es un sistema de cuatro años y el sistema de cacao con especies forestales de 15 años, ambos sistemas agroforestales presentan valores de $19 \mathrm{tm} \mathrm{C}^{-1} /$ ha y $47 \mathrm{tm} \mathrm{C}^{-1}$ respectivamente. Estos valores están por encima de los valores que presentan los sistemas puramente agrícolas de corta duración, lo cual nos demuestra la importancia del establecimiento de éstos sistemas para la recuperación del potencial de captura de Carbono en áreas anteriormente perturbadas por tumba y quema y usados para agricultura. Los sistemas agroforestales pueden recuperar ambientalmente áreas perturbadas y tener un sistema productivo cíclico a corto y mediano plazo, con un adecuado y mejor manejo de las tierras de aptitud forestal.

\section{Flujos de carbono}

Las reservas totales de carbono almacenado nos dan un indicativo del grado de captura de carbono de un sistema, pero no nos especifica la dinámica de éste carbono, por lo cual se ha elaborado los flujos anuales de captura de carbono por hectárea. Estos flujos anuales son los que expresan el dinamismo en acumular el Carbono y es el que la comunidad internacional está considerando para el otorgamiento de créditos por éste servicio ambiental. Cabe destacar que los flujos presentados son un promedio de captura anual en el tiempo de vida del sistema, dado que el crecimiento de una población vegetal no se realiza de manera uniforme en el tiempo, existiendo un mayor crecimiento en los primeros años.

El flujo de carbono en el sistema de bosque secundario de 20 años (3.10 tm $\mathrm{Cha}^{-1}$ año $^{-1}$ ) fue menor en comparación con el del bosque secundario de 50 años (4.69 tm $\mathrm{C} \mathrm{ha}^{-1} \mathrm{año}^{-1}$ ), y esto se debe a las características de éste sistema, el cual fue perturbado y 
descremado, y presenta especies forestales con dap (diámetro a la altura del pecho) menores a $30 \mathrm{~cm}, \mathrm{y}$ con $2.5 \mathrm{~m}^{2}$ de área basal /ha, mientras que en el bosque secundario de 50 años se observa mayor biodiversidad de especies forestales con dap mayores a los $30 \mathrm{~cm}$ totalizando $20 \mathrm{~m}^{2}$ de área basal /ha.

Se puede observar en la Figura 2 que el flujo de carbono del sistema de cacao (3.15 tm/ha/año), es menor que el flujo de carbono del sistema de caféguaba (4.82 $\mathrm{C} \mathrm{ha}^{-1}$ año $^{-1}$ ), esto puede deberse a las edades de los sistemas; el sistema de café tiene una edad de cuatro años, mientras que el sistema de cacao tiene 15 años y como es conocido las capturas de Carbono se deben al incremento de la biomasa anual del sistema y en los primeros años de vida de un sistema este incremento tiene un índice mayor que en los años posteriores, hasta llegar al equilibrio. Los sistemas agroforestales, presentan un flujo de Carbono superior al bosque secundario de 20 años, a pesar de ser los sistemas agroforestales, sistemas plantados; ello podría deberse tanto a la edad de los sistemas como a las perturbaciones al que ha sido expuesto el bosque secundario de 20 años, provocando con ello una alteración al crecimiento regular del sistema.

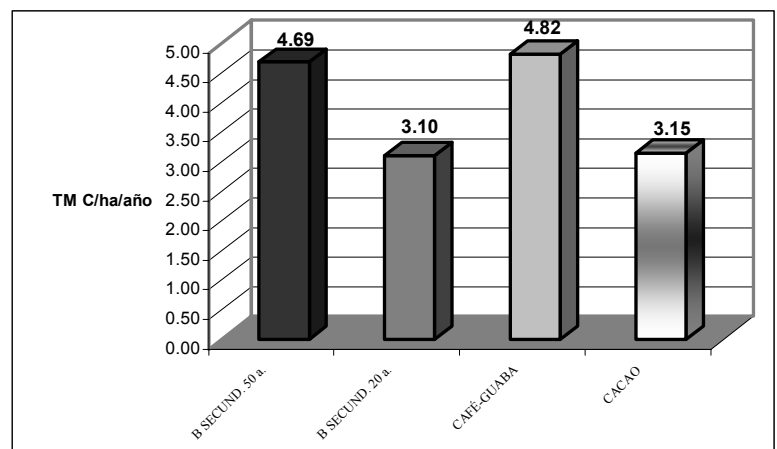

Figura 2 Captura Anual de Carbono por Sistemas.

En las figuras 3.4 y 5 se muestra la captura de Carbono de cada tipo de biomasa aérea evaluada en los sistemas boscosos, en las cuales se puede verificar la importancia de la biomasa arbórea viva, la cual proporciona el mayor porcentaje a la cantidad total de reservas de carbono del sistema, seguido por la biomasa arbórea muerta.

Cabe destacar que en el sistema de bosque primario se encuentra la mayor cantidad árboles parados y caídos muertos, esto probablemente por que el sistema está en un proceso de regeneración continua, motivo por el cual porcentualmente en éste sistema hay menos árboles vivos que en los sistemas de bosque secundario, por lo que se puede deducir que hay una gran variación en la dinámica forestal de éste sistema (la dinámica forestal del sistema varia en: estructura de la población, en estratos, número de individuos de cada especie, y repartición de los

individuos de cada especie en clases de edad) y está abriendo espacio a especies con dap menor. En estas áreas se muestra un incremento en el flujo anual de captura de carbono por la renovación del área e incremento de poblaciones jóvenes y posteriormente de especies forestales por la renovación de las poblaciones, permitiendo que la dinámica forestal, se reactive mediante la gestión de luz por los aclareos naturales.
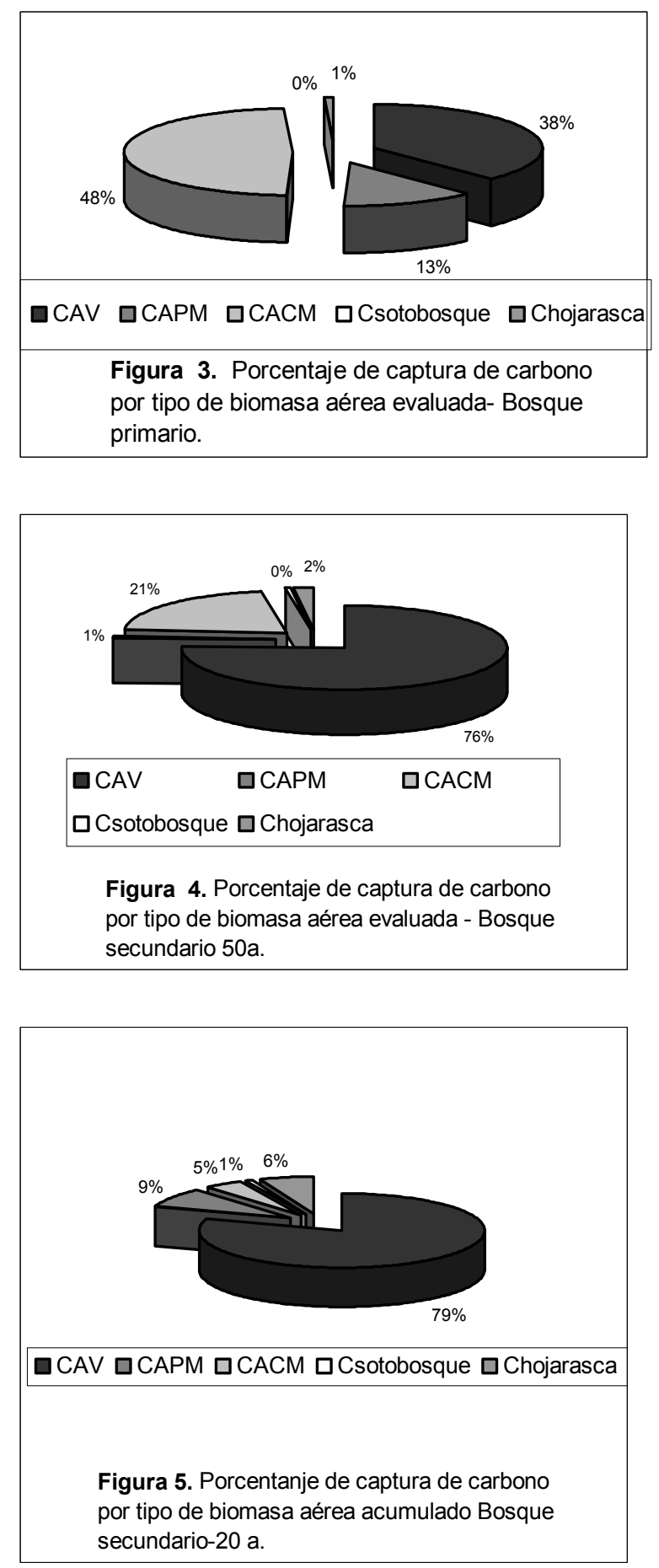
Los niveles de hojarasca y sotobosque, contribuyen muy poco a la captura total de carbono de los sistemas boscosos y sólo fueron relativamente significativos en el bosque secundario de 20 años, esto podría deberse a que el sistema por las perturbaciones continuas no ha logrado alcanzar su nivel real de captura de carbono, de acuerdo a su edad. Cuantitativamente los valores de herbácea y hojarasca de cada sistema boscoso se muestra en la Tabla 1.

Tabla 1. Toneladas de Carbono capturado en biomasa herbácea y hojarasca por hectárea de los sistemas boscosos evaluados.

\begin{tabular}{|l|l|l|}
\hline \multirow{2}{*}{ Sistemas de bosques } & \multicolumn{2}{|c|}{ tm C $\mathrm{ha}^{-1}$} \\
\cline { 2 - 3 } & $\begin{array}{l}\text { Carbono } \\
\text { herbácea }\end{array}$ & $\begin{array}{l}\text { Carbono } \\
\text { hojarasca }\end{array}$ \\
\hline B. Primario & 0.8 & 3.51 \\
\hline B.Secundario 50 a. & 1.03 & 4.7 \\
\hline B.Secundario 20 a. & 0.59 & 3.90 \\
\hline
\end{tabular}

\section{Captura de carbono en cada tipo de biomasa}

En las Figuras 6 y 7, se muestra en porcentajes, las capturas de carbono de cada tipo biomasa aérea evaluada en los sistemas agroforestales. Se puede apreciar que en éstos tipos de sistemas los niveles de hojarasca ayudan significativamente a incrementar el total de reservas de carbono del sistema; esto puede deberse a que en el sistema solo se evalúa tres tipos de biomasa aérea, siendo importante su consideración para la contabilidad total del carbono.

Aunque en cada uno de los sistemas agroforestales, se desarrollan especies diferentes, y tienen edades distintas, es necesario indicar que el nivel de hojarasca en el sistema de café es de $3.98 \mathrm{tm}$ $\mathrm{ha}^{-1} \mathrm{y}$ en el sistema de cacao es de $5.07 \mathrm{tm}$, y estos niveles fueron mayores a los sistemas de bosque secundario. Los niveles de captura de carbono en la biomasa herbácea de éstos sistemas fueron de 0.027 tm C/ha para el café y $0.028 \mathrm{tm} \mathrm{C} \mathrm{ha}^{-1}$ para el cacao, menores a las obtenidas en los sistemas boscosos.

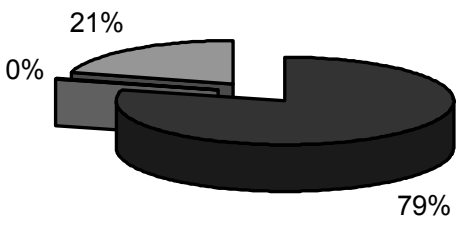

CAV $\square$ Cherbacea $\square$ Chojarasca

Figura 6. Porcentaje de captura de carbono por tipo de biomasa aérea - Sistema de café-guaba.

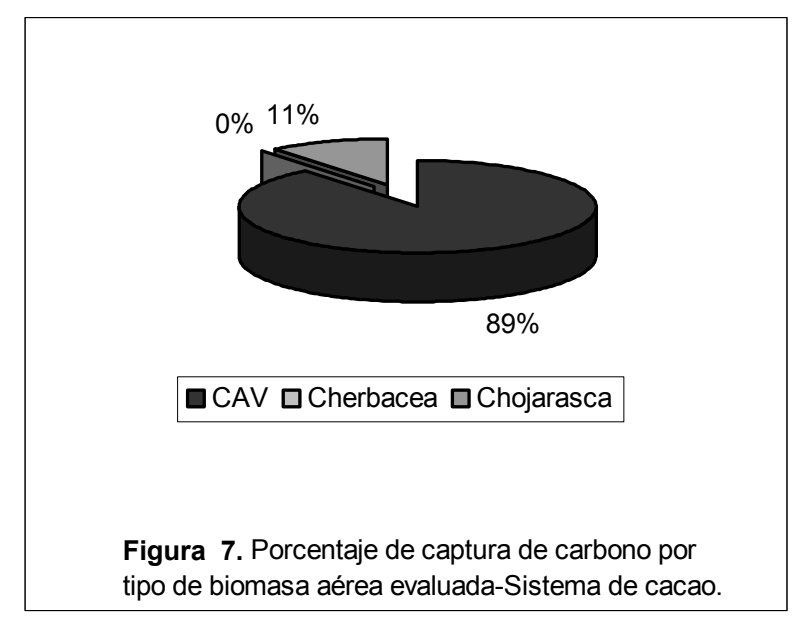

5. Dispersión de las reservas de carbono en cada sistema de uso de la tierra

En las Figuras 8, 9 y 10 se comparan los sistemas con respecto al nivel de reservas de carbono total y la variabilidad que presentan entre los diferentes transectos. En base a esta información y análisis respectivo podemos indicar lo siguiente:

- Los niveles de carbono encontrados en los transectos del bosque primario son bastante altos y la mayor parte sobrepasa los $400 \mathrm{tm} \mathrm{C} \mathrm{ha}^{-1}$, presentándose una alta dispersión entre los transectos debido a la variabilidad innata de éste ecosistema y que también están influenciados por la variabilidad del suelo en que se desarrollan. El Bosque secundario de 50 años, es el siguiente sistema con mayor cantidad de reservas de carbono, presenta también una distribución asimétrica, con una mayor variación con el $50 \%$ de valores cercanos a un rango entre los $300 \mathrm{tm} \mathrm{C}$ ha1 y 400 tm C ha-1 y el $50 \%$ de esos transectos presentan valores muy cercanos a las $200 \mathrm{tm} / \mathrm{ha}$.

- El Bosque secundario descremado de 20 años, presenta distribución asimétrica con el $80 \%$ de sus valores por encima de los $50 \mathrm{tm} \mathrm{C} \mathrm{ha}^{-1}$, solo un valor presenta bastante alto y por encima del promedio, cerca del $94 \mathrm{tm} \mathrm{C} \mathrm{ha}^{-1}$.

- Con relación a los sistemas agroforestales, se puede indicar que no presenta grandes rangos de variación, como se observa en los sistemas de bosque primario y secundario de 50 años. Para el sistema de cacao el $75 \%$ de los valores de las reservas de Carbono se encuentra por encima de los $50 \mathrm{tm} \mathrm{C} / \mathrm{ha}$. Para el sistema de café- guaba el $75 \%$ de los niveles de los transectos se encuentra por encima de las $20 \mathrm{tm} \mathrm{C}^{-1}$. Es significativo el incremento de captura de Carbono en estos sistemas con relación a los sistemas agrícolas.

- Los sistemas agrícolas de maíz y arroz y el sistema de pastos presentan muy poca dispersión debido a la homogeneidad de plantaciones anuales $\mathrm{y}$ 
perennes de corte bajo. La mayor mediana entre los tres sistemas la presenta el sistema de maíz.

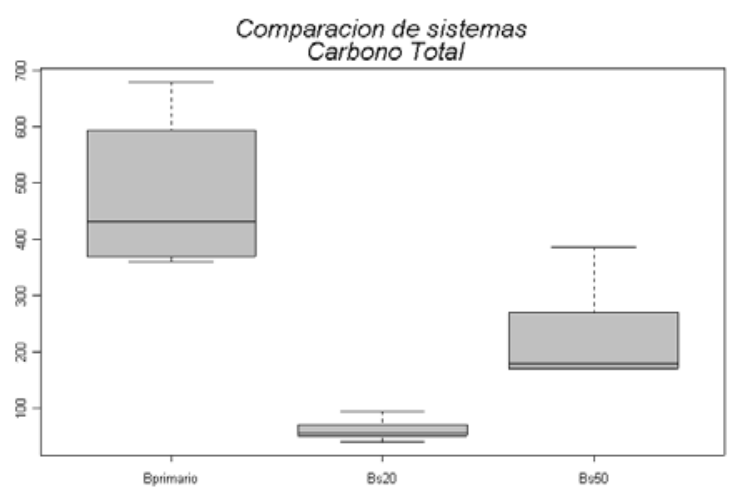

Figura 8. Comparación entre los sistemas boscosos evaluados

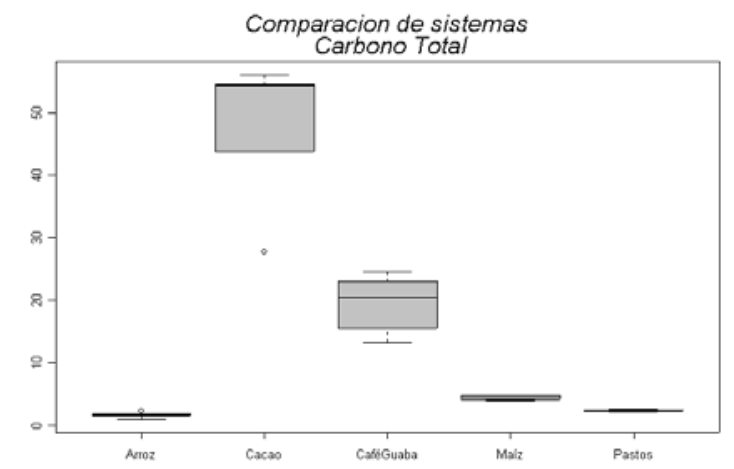

Figura 9 Comparación entre los sistemas agroforestales y agrícolas evaluados.

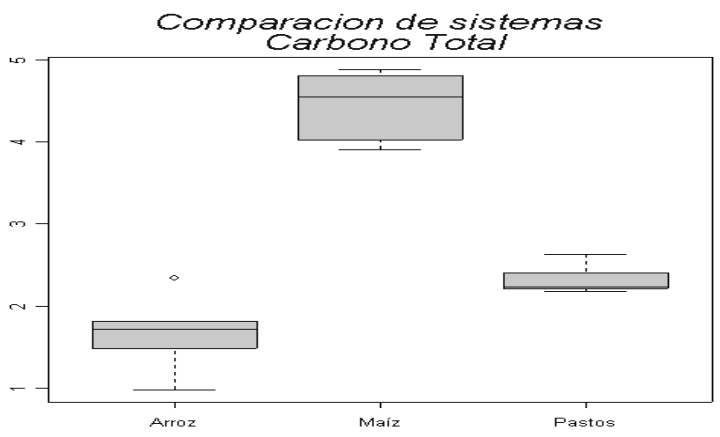

Figura 10. Comparación entre los sistemas agrícolas.

Haciendo una síntesis de lo observado en las Figuras 8, 9, 10, se puede inferir que, mientras mayor complejidad tiene el sistema, más amplio es el rango de variación entre los transectos evaluados, siendo ello observado en los sistemas boscosos, principalmente en bosques sin intervención humana y que tienen una gran dinámica forestal natural.

\section{Climas predominantes en cada localidad}

Para realizar un análisis comparativo entre los resultados obtenidos de la captura de carbono de biomasa aérea en los sistemas evaluados en Yurimaguas y los evaluados en Tarapoto, se realiza primero el análisis de los diferentes climas en éstas áreas:

En Yurimaguas el clima es húmedo tropical, con un régimen térmico casi uniforme en toda la zona, sufriendo un descenso durante los meses de Mayo, Junio y Julio, con temperaturas superiores a los $24{ }^{\circ} \mathrm{C}$, y precipitaciones cuyos promedios oscilan entre los 2 000 y $4000 \mathrm{~mm}$.

En Tarapoto el clima es seco tropical y subhúmedo y húmedo tropical, en el clima seco tropical se presenta mayor tiempo de exposición del sol, con temperaturas promedio superiores a los $24^{\circ} \mathrm{C}$ y precipitaciones en un rango de 1000 y $2000 \mathrm{~mm}$ y en algunos sectores con rangos inferiores a los $1000 \mathrm{~mm}$ (Bosque secundario 20 años, sistemas agrícolas, sistema de pastos y sistema agroforestal de cacao); el clima húmedo subtropical se caracteriza por fluctuación de la temperatura entre $\operatorname{los} 16^{\circ} \mathrm{C}$ y $24{ }^{\circ} \mathrm{C}$, con precipitaciones que oscilan entre los 1100 y 2000 $\mathrm{mm}$ siendo éstas suaves y prolongadas (bosques secundarios de 50), se encuentran ocupando las partes altas, a partir de una elevación aproximada de 700 metros. En el clima húmedo tropical se localizaron las áreas de Bosque Primario y el sistema de café.

La presión de deforestación en la zona Yurimaguas, se ha incrementado en los últimos años especialmente por la apertura de la carretera que une esta localidad con la ciudad de Tarapoto y en la ciudad de Tarapoto la deforestación ha sido tal que solo han quedado pequeñas áreas con difícil acceso; las áreas boscosas evaluadas son estos pequeños vestigios que quedan cercanos a la ciudad de Tarapoto. Con relación a los suelos se puede indicar que en Yurimaguas el suelo es un Ultisol típico, silicio Iso-hipertérmico. La textura de los suelos son franco arenosos con porcentajes de arcilla no mayor de $20 \%$ en los primeros $15 \mathrm{~cm}$ de profundidad. El nitrógeno y la materia orgánica es baja con alta acidez y con bajos niveles de cationes y fósforo y alta saturación de aluminio.

La textura de los suelos de Tarapoto van desde Franco a Franco arcilloso con altos porcentajes de arcilla llegando hasta $35 \%$ en los primeros $20 \mathrm{~cm}$. El pH de estos suelos va desde ácido con niveles de $\mathrm{pH}$ de 4.5 hasta alcalino con $\mathrm{pH}$ de 10 en algunos casos, con porcentaje cero de saturación de aluminio y tienen alto nivel de fósforo y son clasificados como inceptisoles.

Comparativamente se puede indicar de acuerdo a los resultados, lo siguiente:

- El Bosque primario en Tarapoto presenta mayor cantidad de reservas de carbono que los bosques primarios en Yurimaguas, la mayor cantidad de biomasa arbórea en éste sistema, sotobosque y hojarasca son mayores que en bosques primario de Yurimaguas. 
- El Bosque secundario de 50 años, tiene gran cantidad de reservas, y casi se asemeja a las reservas del bosque primario de 100 años de Yurimaguas, esta peculiaridad, puede tener su explicación en el microclima de este sistema el cual es bastante húmedo todo el año, lo que propicia que las especies crezcan y tengan grandes volúmenes.

- El Bosque secundario de 20 años de Tarapoto, no guarda relación con las reservas de los bosques secundarios de Yurimaguas y tiene menores reservas que éstos a pesar que cronológicamente es mayor. Esto puede tener su explicación en que el bosque secundario de Tarapoto es un bosque descremado y en algunas áreas se nota intervención reciente y las especies arbóreas de este sistema no presentan un diámetro mayor a 25 $\mathrm{cm}$ de dap, también es probable que el clima haya influenciado en su desarrollo, ya que el microclima en el bosque secundario de 20 años es seco y con menores precipitaciones.

- El sistema de cacao-15 años de Tarapoto tiene reservas similares al sistema de cacao de Yurimaguas de 20 años, la diferencia entre ambas puede deberse a la edad. Con relación a los sistemas de café-guaba se puede observar que las reservas del sistema de Tarapoto son menores ya que tiene solo cuatro años en comparación de los 10 años del sistema de Yurimaguas. En éste tipo de sistemas la diferencia la otorga el número de especies forestales que se localicen por hectárea en la plantación.

- Con relación a la comparación del Flujo de Carbono entre los sistemas de Yurimaguas y los sistemas de Tarapoto, siguen básicamente el esquema general, donde el flujo de Carbono es mayor en sistemas más jóvenes.

\section{Curvas alométricas}

Se desarrolló la fórmula alométrica para el café, de cuatro años, debido que en investigaciones anteriores se pudo observar que la fórmula utilizada para conocer la biomasa aérea viva en los diferentes sistemas $\left(0.1184 *\right.$ dap $\left.{ }^{2,53}\right)$ no se adecuaba a las características del café. Para el desarrollo de ésta fórmula se realizó el método destructivo de tres a cuatro especies de café por transecto. La mejor correlación se obtuvo entre el diámetro del arbusto y la biomasa seca, siendo su formula la siguiente:

$\mathrm{Y}=0.0474 \mathrm{X}^{2.629}$, donde $\mathrm{Y}$ es la biomasa seca del café y X el diámetro del arbusto.

El método para realizar las curvas alométricas está descrito en el Manual de determinación de las reservas totales de carbono en los diferentes sistemas de uso de la tierra (Arévalo et al., 2003).

\section{Conclusiones}

1. Los sistemas de bosques evaluados (Bosque primario, Bosque secundario 50 años y Bosque secundario de 20 años) tienen los contenidos más altos de Captura de Carbono. Los bosques menos intervenidos presentan mayor cantidad de Carbono. El nivel de Carbono en todos los sistemas agrícolas y agroforestales es mas bajo que el de los bosques naturales.

2. El nivel de Carbono en biomasa aérea de los sistemas agroforestales avaluados (café-guaba, cacao) son más altos que los sistemas agrícolas y los de pastos manejados, teniendo en cuenta esto y los beneficios ambientales de estos tipos de sistemas es recomendable utilizarlos en áreas degradadas.

3. Existe grandes pérdidas de los sumideros de carbono, si se cambian extensiones de bosque por cultivos agrícolas, convirtiéndose éstas áreas en áreas con insignificantes niveles de captura y en algunos casos, áreas que son fuentes de emisión de Gases Efecto Invernadero- GEI.

4. Las retenciones de carbono en los sistemas boscosos evaluados, tienen mayor variabilidad que sistemas agroforestales y agrícolas, de acuerdo al área de evaluación determinada del sistema, por la dinámica y características forestales de las mismas.

5. Los sistemas agroforestales, al combinar los cultivos o frutales con especies forestales, incrementan sus niveles de captura de Carbono, mejorando además su productividad. En el mercado de $\mathrm{CO}_{2}$ equivalente se podría obtener pagos que mejoraría la rentabilidad de la producción y ayudaría a su mantenimiento, con ello el establecimiento de estos sistemas serían más atractivos para las comunidades de diferentes lugares del país.

6. Los cultivos agrícolas no contribuyen significativamente con la retención de carbono. El $\mathrm{CO}_{2}$ atmosférico, uno de los principales gases efecto invernadero, no es sustancialmente retenido en los cultivos, además puede producir fugas de otros GEI, como óxido nitroso, producto de la incorporación de plaguicidas o $\mathrm{CO}_{2}$ por la quema de rastrojos agrícolas; lo cual reduce aún más su captura neta total.

\section{RECOMENDACIONES}

1. Es necesario monitorear el incremento de biomasa y la captura de carbono en plantaciones y en diferentes especies forestales comerciales, así como el desarrollo de fórmulas alométricas de cada una de las especies más utilizadas en los sistemas agroforestales peruanos.

2. Realizar cálculos de retenciones de carbono y $\mathrm{CO}_{2}$ equivalente netos en diferentes sistemas de uso de la tierra, considerando las fugas de $\mathrm{CO}_{2} \mathrm{u}$ 
otros gases efecto invernadero por actividades antrópicas o el uso de plaguicidas, en los mismos.

3. Si en los próximos años se efectuase un pago por captura de carbono en bosques, es recomendable realizar las negociaciones con bosques secundarios, donde el flujo anual de crecimiento en la biomasa es mayor que en los bosques adultos, que casi ya han llegado al equilibrio.

4. Una opción para recuperar las áreas deforestadas que están en proceso de degradación, podrían ser los sistemas agroforestales, que pueden almacenar cantidades adicionales de carbono. Así mismo desarrollar tecnologías agroforestales ecológicamente sostenibles para los agricultores de bajos recursos y el aumento del bienestar de la población que vive dentro y alrededor de la foresta y otros ecosistemas.

5. Se debe establecer y mantener programas nacionales con diferentes ecosistemas, con una base estratégica de investigación, que además de desarrollar sistemas agroforestales sostenibles para el trópico húmedo y semi-árido, en el que se incluya la zona andina con ecosistemas de montañas; se tenga la capacidad de evaluar los aspectos medio ambientales como el secuestro de carbono y la emisión de gases efecto invernadero.

6. La metodología utilizada para la estimación de carbono, puede adecuarse para conocer el carbono almacenado en plantaciones forestales, y agroforestería y estimar con ello el pago por servicios ambientales de captura de carbono que debería negociarse.

\section{Agradecimientos}

Agradezco la colaboración financiera de "The World Agroforestry Centre", al Instituto de Cultivos Tropicales de Tarapoto por el servicio de su laboratorio $\mathrm{y}$ al Instituto Nacional de Recursos Naturales por el tiempo que me otorgó para la realización de la etapa de campo. Así mismo agradezco la colaboración al Ing. Roque Fernández, Ing. Piero del Pozo del INRENA y al Señor Aniceto Daza del Herbario Forestal de la Universidad Nacional Agraria.

\section{Literatura citada}

Alegre J., Arevalo L., Ricse A., Callo-Concha \& Palm C. 2004. Secuestramiento de carbono con sistemas alternativos en el Peru In. Sistemas Agroflorestais, Tendencia da Agricultura Ecologica nos Tropicos, sustento da vida e sustento de vida. : 27-32 Editores: M. Muller, A. Gama-Rodrigues, I. Silva Fontes \& M. Carvalho. Sociedadee Brasileira de sistemas Agroflorestais, Comissao Executiva do plano da lavoura cacauria y Universidade Estadual do Norte Fluminense .Ilehues- BA.

Arévalo L., Alegre J.C. \& Palm C. 2003. Manual de reservas de carbono en diferentes Sistemas de uso de la tierra. Edición: Grafica Miguel Alvarez.

Dixon R.K. 1995. Agroforestry systems: sources or sinks of greenhouse gases?. Agroforestry Systems. 31: 99-116.

Douglas C.M. 1991. Design and Análisis of Experiments, ed.John Wiley and Sons New York.

Gralb H. G., Hupfer P. \& Lozán J.L. 2001.El efecto Invernadero Antropógeno En: El Clima en el siglo XXI: Introducción al Problema Global del Clima más protección climática-menos riesgos para el futuro.

Juran J.M. \& Gryna F.M. 1988. Juran's Quality Control Hand book $4^{\circ}$ ed. Mc Graw- Hill.

Palm C. A., Tomich T., van Noordwijk M., Vosti S., Gockowski J., Alegre J. \& Verchot L. 2004. Mitigating GHG emissions in the humid tropics: Case studies from the Alternatives to Slash and Burn Program (ASB). Environment, Development and Sustainability. 6: 145-162.

Woomer P.L., Palm C.A., Alegre J., Castilla C., Cordeiro D.G., Hairiah K., Kotto-Same J., Moukam A., Ricse A., Rodrigues V. \& van Noordwijk M. 2000. Slash-and-burn effects on carbon stocks in the humid tropics. Edited by In: R. Lal, J.M. Kimble and B.A. Stewart .Global Climate Change and Tropical Ecosystems.: 99115. CRC Press, Inc. Boca Raton, FLa. USA.

\footnotetext{
${ }^{1}$ Instituto Nacional de Recursos Naturales (INRENA), Perú, e-mail tlapeyre@mtc.gob.pe

${ }^{2}$ Universidad Agraria la Molina (UNALM) Perú, e-mail: J.alegre@cgiar.org

${ }^{3}$ Proyecto Cordillera Azul (CIMA) Tarapoto, Perú. e-mail: larevalo@cima-cordilleraazul.org.pe
} 